\title{
Development of Nanoparticulate Hydrogel Loaded with Crossandra infundibuliformis Extract
}

\author{
Divya Jyothi* ${ }^{*}$, Sneh Priya ${ }^{2}$, Jainey P James ${ }^{3}$ \\ 'Department of Pharmacognosy, Nitte Gulabi Shetty Memorial Institute of Pharmaceutical Sciences, Deralakatte, Karnataka, INDIA. \\ 2Department of Pharmaceutics, Nitte Gulabi Shetty Memorial Institute of Pharmaceutical Sciences, Deralakatte, Karnataka, INDIA. \\ ${ }^{3}$ Department of Pharmaceutical Chemistry, Nitte Gulabi Shetty Memorial Institute of Pharmaceutical Sciences, Deralakatte, Karnataka, INDIA.
}

\begin{abstract}
Objective: Present study is aimed at formulation of hydrogel containing PLGA nanoparticles loaded with of Crossandra infundibuliformis (Cl) extract. Leaf extract of Crossandra infundibuliformis has been reported to possess antibacterial, antifungal activity hence an attempt was done to improve the efficacy of the extract by formulating into nanoparticulate hydrogel which can be used as a feasible alternative to conventional formulations of Crossandra infundibuliformis extract with dual benefit of sustained release and advanced permeation characteristics for transdermal application. Methodology: In this study, the active ingredients present in the leaves of Crossandra infundibuliformis (Cl) were extracted by cold maceration using ethanol. The crude extracts were developed into polymeric nanoparticles by nanoprecipitation method using PLGA as polymer, polyvinyl alcohol as surfactant and dimethyl sulphoxide as organic phase solvent. Polymeric nanoparticles were incorporated into gel matrix containing HPMC K4M as gel matrix base. Results: Polymeric nanoparticles showed nearly spherical shape with z-average $143-325 \mathrm{~nm}$, PDI in the range of $0.235-0.299$ and $-2.08 \mathrm{mV}$ to $-3.58 \mathrm{mV}$ zeta potential with maximum $\%$
\end{abstract}

drug entrapment of $69 \%$. Nanoparticulate hydrogel formulations showed high viscosity, neutral $\mathrm{pH}$ with good spreadability which is suitable for transdermal application. In vitro drug release showed initial burst release of $28.56 \pm 0.93 \%$ with prolonged drug release of $90.06 \pm 0.93 \%$ from optimized formulation up to $24 \mathrm{~h}$. Conclusion: Nanoparticulate hydrogel can be used as carrier for transdermal delivery of extract of Crossandra infundibuliformis.

Key words: Crossandra, Nanoparticle, Hydrogel.

Correspondence:

Divya Jyothi,

Department of Pharmacognosy, Nitte Gulabi Shetty Memorial Institute of Pharmaceutical Sciences, Nitte University, Deralakatte, Karnataka, INDIA.

Phone: +918722821164

E-mail: divya.jyothi84@gmail.com

DOI: 10.5530/jyp.2017.9.15

\section{INTRODUCTION}

Herbal medicines have been extensively used around the world since ancient times. The efficacy of medicinal plants depends on the supply of active constituents. It has been widely proposed to combine herbal medicine with nanotechnology, because nanostructured systems might be able to potentiate the action of plant extracts, reducing the required dose and side effects, and improving activity. ${ }^{1}$ Nanosystems can deliver the active constituent at a sufficient concentration during the entire treatment period, directing it to the desired site of action. Conventional treatments do not meet these requirements.

Polymeric nanoparticles are made from biodegradable and biocompatible polymers, represent an option for controlled drug delivery and are promising formulation used for drug delivery systems, because they can be targeted where as hydrogels are polymeric networks with threedimensional configuration capable of imbibing high amounts of water or biological fluids. ${ }^{2,3}$ Hydrogel nanoparticles have gained considerable attention in recent years as one of the most promising nanoparticulate drug delivery systems owing to their unique potentials via combining the characteristics of a hydrogel system (e.g., hydrophilicity and extremely high water content) with a nanoparticle (e.g., very small size). Crossandra infundibuliformis belonging to family Acanthaceae is observed to show several medicinal properties and especially, its leaf extract was reported to have the antioxidant, antibacterial, antifungal, anticandidal and larvicidal activity. ${ }^{4-7}$ These activites may be due to the presence of phytoconstituents such as Carbohydrates, Flavanoids, Alkaloids, steroids, tannins, terpenoids, Saponins which was reported in the extracts of C. infundibuliformis leaves from the phytochemical analysis. ${ }^{8}$ Leaf extract was also found to effective topically for wound healing. ${ }^{9}$ To make the extract into efficient candidate for topical application, study was conducted to develop nanoparticulate hydrogel formulation containing CI extract which is expected to have sustained release as well as improved permeation characteristics due to nano size and hydrophilic nature.

\section{MATERIALS AND METHODS}

\section{Materials}

Poly Lactic Glycolic Acid (PLGA 50:50) was procured from Sigma Aldrich, Bangalore and H.P.M.C K4M was obtained from Yarrow Chem products, Mumbai.

\section{Preparation of plant extract and phytochemical analysis}

Leaves of Crossandra infundibuliformis were collected from local area of Bantwal Taluk and authenticated by Prof (Dr) Nagalakhamma St. Aloysius College, Mangalore. Leaves were dried, powdered and then extracted by cold maceration method using $95 \%$ ethanol as solvent for 7 days. After the extraction solution was filtered and filtrate was evaporated to dryness and percentage yield was calculated.

Prepared extract was subjected to different chemical tests according to standard procedure in order to determine the presence of various phytoconstituents. ${ }^{10}$ 


\section{Calibration of Crossandra infundibuliformis extract by UV-Visible Spectrophotometer}

The ethanolic extract was dissolved in small amount of ethanol, followed by addition of phosphate buffer of $\mathrm{pH} 6.8$ and then suitably diluted. The solutions were scanned for its specific UV-Visible range of absorbance maxima. Then the absorbance of the different serial diluted samples was measured at the $\lambda$ max, and a standard calibration curve was plotted with concentration against absorbance.

\section{Preparation of Polymeric Nanoparticles}

The nanoparticles of herbal extract with PLGA as polymer were prepared using emulsion solvent evaporation (nanoprecipitation) method. ${ }^{11}$ During the process, the organic phase was prepared by dissolving accurately weighed PLGA and plant extract in DMSO as organic solvent. The organic phase was then added dropwise at the rate of $1 \mathrm{ml} / \mathrm{min}$ into an aqueous phase containing surfactant (PVA - $0.5 \%$ ) dissolved in water as aqueous solvent. The nanoparticles suspension was kept under continuous stirring at $300 \mathrm{rpm}$ for $3 \mathrm{~h}$ at $30^{\circ} \mathrm{C}$ to allow the complete evaporation of DMSO, leaving behind the colloidal suspension of PLGA nanoparticles containing plant extract in aqueous phase. The colloidal nanosuspension was centrifuged at 12,000 rpm (Remi, Mumbai, India) for $30 \mathrm{~min}$ at $4^{\circ} \mathrm{C}$ to get the final nanoparticulate containing pellet as encapsulated plant extract. The pellet was washed with deionized water twice to remove unentrapped drug from the surface of nanoparticles. Nanoparticulate pellets were redispersed in water. Formulation ingredients of nanoparticles are given in Table 1.

\section{Characterization of Nanoparticles Measurement of Particle size of the nanoparticles}

Average particle size (z-average) and Polydispersity index (PDI) of the developed nanoparticles were determined by laser dynamic light scattering using Malvern Zetasizer (Nano ZS, Malvern Instruments, $\mathrm{UK})$. The PDI value indicates the particle size distribution of nanoparticles in a given sample.

\section{Measurement Zeta Potential}

Zeta potential of nanoparticles loaded with extract was measured using Malvern Zetasizer (Nano ZS, Malvern Instruments, UK). Zeta potential indicates the surface charge on the particles and was measured to determine the stability of nanoparticles in the suspension. ${ }^{12}$

\section{Entrapment efficiency of the polymeric herbal nanoparticle}

The nanoparticle suspension formulated with the extract and polymer was ultra centrifuged at $18,000 \mathrm{rpm}$ for 30 minutes in a cooling centrifuge apparatus and then the supernatant solution was diluted suitably to measure the absorbance, from which the concentration of drug in supernatant was calculated using the standard calibration data. ${ }^{13}$ The entrapment efficiency of the extract in the polymeric nanoparticles was calculated using the formula,

$$
\text { Entrapment Efficienty (\%) }=\frac{\begin{array}{l}
\text { Total amount of Drug } \square \\
\text { Amount of Drug is supermatant }
\end{array}}{\text { Total amount of Drug added }} \square 100
$$

Based on the results of particle size analysis, PDI and entrapment efficiency, optimised formulation of nanoparticles was chosen and it was subjected for scanning electron microscopy and transmission electron microscopy.

\section{Scanning electron microscopy (SEM)}

Shape and surface morphology of the nanoparticles were studied using SEM (JEOL, JSM 50A, Tokyo, Japan). An appropriate amount of colloidal dispersion of polymeric nanoparticle was mounted onto metal (aluminium) using double-sided adhesive tape and fractured with a razor blade. The samples were sputter-coated with gold/palladium for $120 \mathrm{sec}$ at $14 \mathrm{~mA}$ under argon atmosphere for secondary electron emissive SEM and observed for morphology, at acceleration voltage of $15 \mathrm{KV}$.

\section{Transmission Electron Microscopy (TEM)}

The morphology of formulation was observed under TEM.

\section{Formulation of Nanoparticulate Hydrogel}

Prepared nanoparticles (N1-N3) were found in nanosize range and therefore incorporated in gel matrix. Hydroxypropyl methylcellulose K4m (HPMC K4M), was selected as gel matrix base. Required quantity of gelling agent was weighed and dispersed in a small quantity of distilled water to form a homogeneous dispersion. Prepared nanoparticle formulation was added to the above solution to obtain hydrogel formulation NG1, NG2, NG3. Other excipients (methyl paraben and propyl paraben) were also added with stirring. The $\mathrm{pH}$ values were subsequently regulated to 6-9 by using triethanolamine.

\section{Characterization of Hydrogel Formulations Determination of drug content}

The amount of drug contained in the prepared hydrogel was determined by dissolving $100 \mathrm{mg}$ of prepared hydrogel in acetone and diluted using phosphate buffer of $\mathrm{pH}$ 6.8. This mixture was analysed UV spectrophotometer at $203 \mathrm{~nm}$ against phosphate buffer as blank.

\section{pHDetermination}

The $\mathrm{pH}$ of the formulation was determined at ambient temperature with digital $\mathrm{pH}$ meter (digital $\mathrm{pH}$ meter, 335, systronics, Noroda, Ahmedabad). ${ }^{14}$ Since the formulation was a topical formulation to be applied to the skin, therefore $\mathrm{pH}$ measurement was essential to ensure non irritating nature of the formulation.

\section{Determination of Spreadability}

The spreadability of prepared hydrogel formulation was determined $48 \mathrm{~h}$ after preparation by measuring the spreading diameter of formulation between the two glass plates after $1 \mathrm{~min}$. A weight of $500 \mathrm{mg}$ of hydrogel was placed within a circle of diameter $1 \mathrm{~cm}$ premarked on glass plate over which a second glass plate was placed. The increase in diameter as a consequence of weights added leading to spreading of gel was noted. ${ }^{15}$

\section{Measurement of Viscosity}

The viscosity of the prepared formulations was determined at different angular velocities at $32.0 \pm 0.1^{\circ} \mathrm{C}$ using spindle number 4 (Brookfield DV-II+ Pro viscometer). ${ }^{16}$

\section{In vitro Drug Release Study of Hydrogel}

Prepared nanoparticulate hydrogel formulations (NG1, NG2, NG3) was evaluated for the percentage of release of the extract constituents, for 24 hours. In vitro release of drug across the dialysis bag $(12 \mathrm{Kda}, \mathrm{Hi}$ Media) soaked in deionized water for $12 \mathrm{~h}$ before use was performed by using diffusion cell (containing $1 \mathrm{ml}$ of sample) and $80 \mathrm{ml}$ of phosphate buffer $\mathrm{pH} 6.8$ as the dissolution medium $(\mathrm{n}=6)$. The dissolution medium was maintained at $37 \pm 0.5^{\circ} \mathrm{C}$ and the medium was stirred at $100 \mathrm{rpm}$ with the help of small teflon coated magnetic bead. Aliquots of the medium were withdrawn at suitable time interval and were replaced with the same volume of fresh medium to maintain the sink condition. These samples were filtered through $0.45 \mu \mathrm{m}$ membrane filter and the collected samples were analyzed using UV-visible spectrophotometer at the Imax of $203 \mathrm{~nm}$. 


\section{RESULTS AND DISCUSSION}

Ethanolic extract of Crossandra infundibuliformis were greenish in colour and percentage yield was found to be $14 \%$ respectively. The preliminary phytochemical analysis showed the presence of flavonoids, tannins, steroids, carbohydrates, proteins in the extract.

\section{Calibration of the crude extract}

The crude ethanolic extract of Crossandra infundibuliformis was dissolved in little amount of ethanol and diluted with phosphate buffer of $\mathrm{pH} 6.8$ and scanned in a UV-spectrophotometer have shown the maximum absorbance wavelength at $203 \mathrm{~nm}$. The serially diluted samples of the extract exhibited the absorbance value in the linearity range and their regression was found to be 0.998 (Figure 1).

\section{Preparation and Characterisation of Polymeric Nanoparticles}

The results of entrapment, size and charge of the nanoparticles are shown in Table 2, where N1, N2, N3 are nanoparticles of Crossandra infundibuliformis ethanolic extract with $50 \mathrm{mg}, 100 \mathrm{mg}$ and $150 \mathrm{mg}$ PLGA respectively.

\section{Particle size and zeta potential of nanoparticles}

The average size of the synthesised nanoparticles was found to lie within the range of 143-325 $\mathrm{nm}$. Size range of polymeric nanoparticles as 100$1000 \mathrm{~nm}$ could provide stable colloidal dispersion. ${ }^{10}$ Polydispersibility index (PDI) of formulations was found to be in a range of 0.235- 0.299. The low PDI values indicated that nanoparticles size was uniform within each formulation. Normally Higher value of PDI indicates the distribution of nanoparticles with variable size range which results in the formation of aggregates and could result in low stability of particle suspension and low homogeneity. Surface charge measured in terms of zeta potential plays a major role for nanoparticles stability as the standard criteria. The zeta potential of the synthesized herbal nanoparticles was found to be $-2.08 \mathrm{mV}$ to $-3.58 \mathrm{mV}$. Zeta potential greater than $+25 \mathrm{mV}$ and less than $-25 \mathrm{mV}$ correlate to higher stability of the nanoparticles. At larger zeta potentials, colloidal nano dispersions are expected to be stable as the charged droplets within them repel one another more strongly, thus overcoming the natural tendency to aggregate which was concluded from the results of zeta potential.

\section{Entrapment efficiency}

Amount of extract entrapped in the polymeric nanoparticles determined using UV-Visible spectrophotometer showed that the extract of different nanoparticle formulation had higher entrapment efficiency in the range of $62-69 \%$. There was no significant difference observed in the formulations prepared with different concentrations of the polymer, since the surfactant level, amount of extract used and all other process variables were kept constant during the nanoparticles development.

Based on results of particle size analysis, PDI and entrapment efficiency, nanoparticle formulation N1containing Crossandra infundibuliformis ethanolic extract with $50 \mathrm{mg}$ of PLGA polymer was selected as optimised formulation as it has nanosize range particles with low PDI and high entrapment efficiency.

\section{Scanning electron microscopy (SEM)}

Shape and surface morphology of the nanoparticles in Optimised formulation N1 was studied using SEM. The SEM study reveals that polymeric nanoparticles were spherical in shape with an average particle size around $143.9 \mathrm{~nm}$ as shown in Figure 2.

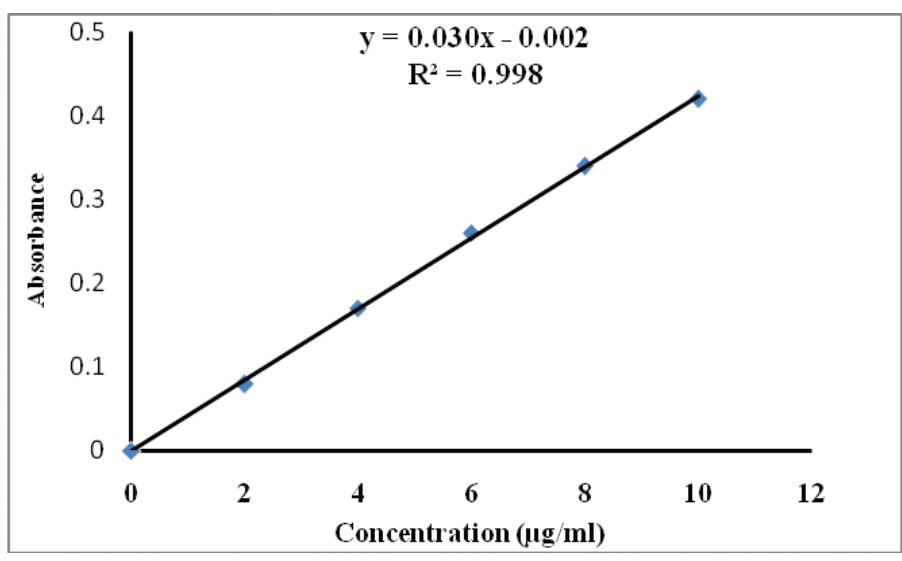

Figure 1: Calibration curve of ethanolic extract of Crossandra infundibuliformis.

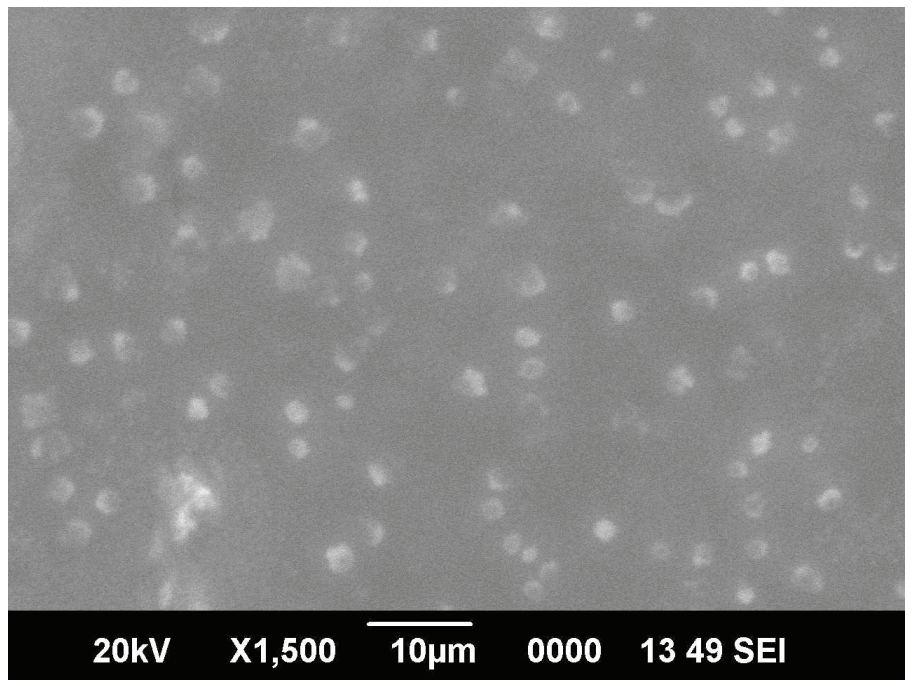

Figure 2: SEM image of optimized formulation loaded with extract of Crossandra infundibuliformis (N1).

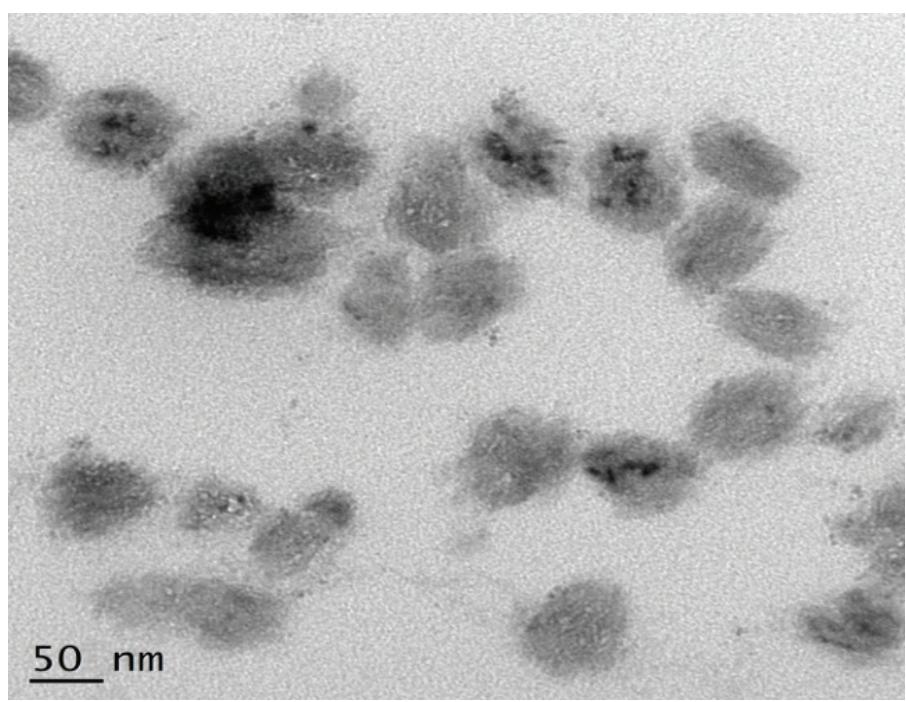

Figure 3: TEM image of optimized formulation of nanoparticles loaded with extract. 


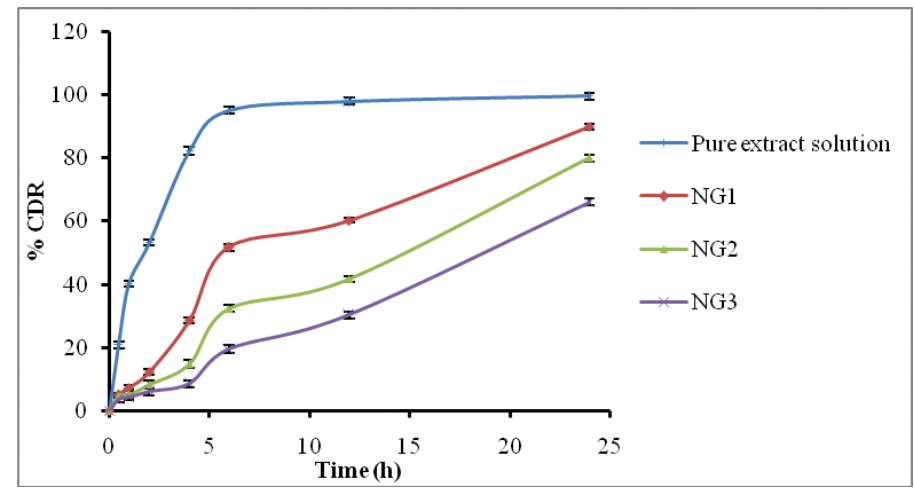

Figure 4: In-vitro drug release profile of nanoparticulate hydrogel loaded with extract of Crossandra infundibuliformis.

Table 1: Formulation ingredients of nanoparticles

\begin{tabular}{cccc}
\hline Formulation code & Extract $(\mathrm{mg})$ & PLGA $(\mathrm{mg})$ & PVA $(\%)$ \\
\hline N1 & 25 & 50 & 0.5 \\
N2 & 25 & 100 & 0.5 \\
N3 & 25 & 150 & 0.5 \\
\hline
\end{tabular}

Table 2: Physicochemical characterization of nanoparticles loaded with extract of Crossandra infundibuliformis

\begin{tabular}{ccccc}
\hline $\begin{array}{c}\text { Formulation } \\
\text { code }\end{array}$ & $\begin{array}{c}\text { Particle Size } \\
(\mathrm{nm})\end{array}$ & PDI & $\begin{array}{c}\text { Zeta Potential } \\
(\mathrm{mV})\end{array}$ & $\begin{array}{c}\text { Entrapment } \\
\text { Efficiency (\%) }\end{array}$ \\
\hline N1 & 143.9 & 0.235 & -2.08 & 68.5 \\
N2 & 283.8 & 0.267 & -3.24 & 65.3 \\
N3 & 321.8 & 0.299 & -3.58 & 62.9 \\
\hline
\end{tabular}

Table 3: Characterization of Hydrogel

\begin{tabular}{ccccc}
\hline $\begin{array}{c}\text { Formulation } \\
\text { code }\end{array}$ & $\begin{array}{c}\text { Drug } \\
\text { content }\end{array}$ & $\mathrm{pH}$ & $\begin{array}{c}\text { Spreadability } \\
(\mathbf{c m})\end{array}$ & $\begin{array}{c}\text { Viscosity } \\
(\mathrm{m} . \mathrm{PaS})\end{array}$ \\
\hline NG1 & $97.8 \pm 0.23$ & $6.7 \pm 0.2$ & $6.0 \pm 0.32$ & $4312 \pm 0.21$ \\
NG2 & $96.4 \pm 0.21$ & $6.6 \pm 0.3$ & $5.5 \pm 0.22$ & $4325 \pm 0.18$ \\
NG3 & $96.6 \pm 0.38$ & $6.7 \pm 0.1$ & $5.8 \pm 0.42$ & $4350 \pm 0.16$ \\
\hline
\end{tabular}

All the values are expressed as mean \pm SD $(n=3)$.

\section{Transmission electron microscopy (TEM)}

The transmission electron microscope revealed a positive image in which nanoparticles appeared dark with bright surroundings as shown in Figure 3. The particle size of sample was less than $1000 \mathrm{~nm}$. These results confirmed that the prepared nanoparticles had nanosize range. Moreover, $\mathrm{z}$-average gives the hydrodynamic size when the particles are suspended in aqueous media. TEM images would give a better understanding of the real geometric size of the particles.

\section{Formulation and characterisation of nanoparticulate Hydrogel formulation}

Hydrogel formulations NG1, NG2, NG3 were prepared by incorporating the nanoparticle formulation N1, N2, N3 into HPMC K4M gel matrix respectively. Prepared hydrogel were subjected for determination of $\mathrm{pH}$, spreadability, viscosity and drug content and results of which is shown in Table 3.

The drug content of nanoparticulate hydrogel formulation was in the range of $96.6 \pm 0.38 \%$ to $97.8 \pm 0.23 \%$. The results showed that the drug was uniformly distributed throughout the formulation and drug loss was minimum while formulating nanoparticulate hydrogel. The $\mathrm{pH}$ values of different nanoparticulate hydrogel formulations were found to be in a range of 6.6-6.7 (nearly neutral), permitting the use of the formulation on the skin. The spreadability of the all formulations exhibited slip and drag phenomenon with higher diameters.

\section{Release studies from nanoparticulate hydrogel formulation}

Release of the extract from the nanoparticulate hydrogel formulation prepared with nanoparticles containing different concentrations of PLGA were compared (Figure 4). Hydrogel formulation NG1 containing nanoparticles loaded with extract of Crossandra infundibuliformis and $50 \mathrm{mg}$ of PLGA, have shown the highest release of $90 \%$ at the end of $24 \mathrm{~h}$ and when the PLGA composition was increased to $100 \mathrm{mg}$ and $150 \mathrm{mg}$ in NG2 and NG3 formulation respectively, the drug release was found to decrease to $80 \%$ and $66 \%$ due to the higher PLGA polymer encapsulation. Hence drug release study indicates that PLGA concentration is known to play an important role in controlling particle size along with release of drug from the matrix. At lower concentration of PLGA in NG1 (50 mg) have smaller particle size compare to NG2(100mg) and NG3(150 mg) therefore showing higher and prolonged drug release. ${ }^{17,18}$ The particles of nanosize range led to a shorter average diffusion path for the matrix-entrapped drug molecules, which also causes faster diffusion. The amount of cumulated drug released from optimised nanoparticulate hydrogel formulation NG1 over $24 \mathrm{~h}$ was $90.06 \%$ where as drug release from the extract solution were found to be $95.13 \%$ within $6 \mathrm{~h}$ and reached $99.66 \%$ by $24 \mathrm{~h}$. Thus, it was clear that incorporation of extract of Crossandra infundibuliformis in PLGA nanoparticles could significantly prolong the release of active constituents.

\section{CONCLUSION}

The novel nanoparticulate hydrogel formulation containing plant extract with suitable viscosity was successfully formulated for transdermal application. PLGA nanoparticles loaded with Crossandra infundibuliformis incorporated into the hydrogels showed controlled release of active constituents which will be a promising carrier for transdermal delivery of active constituents in the extract.

\section{ACKNOWLEDGMENT}

I acknowledge the Nitte University for providing the financial support for performing this project. I am thankful to the authorities of NGSM Institute of Pharmaceutical Sciences for providing the facilities to carry out the work. I am also grateful to Dr. Nagalaxmi, Botany Department, Aloysius College, Mangalore for assisting in the identification of plant material.

\section{CONFLICT OF INTEREST}

No conflict of interest are declared.

\section{ABBREVIATION USED}

nm: Nanometer; mV: Millivolt; PLGA: Poly Lactic Glycolic Acid; DMSO: Dimethyl sulphoxide; HPMC: Hydroxy propyl methyl cellulose; PDI: Polydispersibilty index; mg: Milligram; cm: Centimeter; SEM: Scanning electron Microscopy; PVA: Ploy Vinyl Alcohol; m.PaS: Milli Pascal. 


\section{REFERENCES}

1. Ankita P, Govind P. Usefulness of nanotechnology for herbal medicine. Plant Archiev. 2013;13(2):617-621.

2. Deepak Y, Suruchi S, Anis AC, Sikender M, Hemant, Nadeem BM, et al. Novel approach: Herbal remedies and natural products in pharmaceutical science as nano drug delivery system. Int J Pharmacy Technol. 2011;3(3):3092-3116

3. Juergen S, Ronald S, Michael R. Fundamentals and Applications of Controlled Release Drug Delivery, Eds. Spinger, New York, NY, 2012;75-106.

4. Sharmila N, Gomathi N. Antibacterial, antioxidant activity and phytochemica studies of Crossandra infundibuliformis extract. Int J Phytomed. 2011;3:151-6.

5. Elamathi R, Deepa T, Kavitha R, Kamalakannan P, Sridhar S, Kumar SJ. Phytochemical screening and antimicrobial activity of leaf extracts of Crossandra infundibuliformis $L$ nees on common bacterial and fungal pathogens. Int J Curr Sci. 2011;1:72-7.

6. Madhumitha G, Saral AM. Preliminary phytochemical analysis, antibacterial, antifungal and anticandidal activities of successive extracts of Crossandra infundibuliformis. Asian Pac J Trop Med. 2011;4(3):192-5.

7. Madhumitha G, Saral AM. Screening of larvicidal activity of Crossandra infundibuliformis extract against Anopheles stephensi, Aedes aegypti and Culex quinquefasciatus. Int J Pharm Pharm Sci. 2012;4(1):485-7.

8. Vadivel E, Panwal SV. In vitro anticancer and insecticidal activity of Crossandra infundibuliformis. J Chem Pharm Res. 2016:8(5):260-264

9. Sumalatha K. Wound healing process and effect of petroleum ether extract of Crossandra infundibuliformis leaf extract in excision wound model. Int $J$ Preclinical and Pharmaceutical Res. 2012;3(1):42-9.
10. Kokate CK, Purohit AP, Gokhale SB. Pharmacognosy. 10 $0^{\text {th }}$ ed. New Delhi: Nirali Prakashan Pvt Ltd.; 1998;92-4.

11. Sharma D, Maheshwari D, Philip G, Rana R, Bhatia S, Singh M et al. Formulation and optimization of polymeric nanoparticles for intranasal delivery of lorazepam using box-behnken design: In vitro and in vivo evaluation. BioMed Research International 2014; http://dx.doi.org/10.1155/2014/156010.

12. Renugadevi K, Inbakandan D, Bavanilatha M, Poornima V. Cissus quadrangularis assisted biosynthesis of silver nanoparticles with antimicrobial and anticancer potentials. Int J Pharm Bio Sci. 2012;3:437-45.

13. Ravi KV, Sevukarajan M, Vulava J, Pavankumar AG, Deepthi Y, Manjunath M, et al. Improvement of dissolution characteristics and bioavailability of Tadalafil by solid dispersion technique using water-soluble polymers. Int J Adv Pharmaceutics. 2012;2:56-63.

14. Mishra US, Murthy PN, Pasa G, Kumar S. Formulation development and standardisation of herbal gel containing methanolic extract of Butea frondosa. Int Res J Pharm. 2011;2(11):126-9.

15. Khan AW, Kotta S, Shahid Ansari SH, Sharma RK, Kumar A, Ali J. Formulation development, optimization and evaluation of aloe vera gel for wound healing. Pharmacog Mag. 2013;9(1):1-22.

16. Basha BN, Prakasam K, Goli D. Formulation and evaluation of gel containing fluconazole - antifungal agent. Int J Drug Dev Res. 2011;3(4):109-28.

17. Seju U, Kumar A, Sawant KK. Development and evaluation of olanzapine-loaded PLGA nanoparticles for nose-to brain delivery: in vitro and in vivo studies. Acta Biomaterialia. 2011;7(12):4169-76.

18. Kalimouttou S, Skiba M, Bon P, Dechelotte P,ArnaudP, Lahiani-Skiba M. SinefunginPLGA nanoparticles: drug loading, characterization, in vitro drug release and in vivo studies. J Nanosci \&Nanotechnol.2009;9(1):150-158.

Article History: Submission Date: 13-07-16; Revision Date: 21-08-16; Accepted Date: 02-09-16.

Cite this article: Jyothi D, Priya S, James JP. Development of Nanoparticulate Hydrogel Loaded with Crossandra infundibuliformis Extract. J Young Pharm. 2017:9(1):78-82. 